\title{
TITLE:
}

\section{Explicit solutions of the classical Calogero and Sutherland systems for any root system}

\author{
$\operatorname{AUTHOR}(\mathrm{S})$ :
}

Sasaki, R; Takasaki, K

\section{CITATION:}

Sasaki, R ...[et al]. Explicit solutions of the classical Calogero and Sutherland systems for any root system. JOURNAL OF MATHEMATICAL PHYSICS 2006, 47(1): 012701.

\section{ISSUE DATE:}

2006-01

URL:

http://hdl.handle.net/2433/50460

\section{RIGHT:}

Copyright 2006 American Institute of Physics. This article may be downloaded for personal use only. Any other use requires prior permission of the author and the American Institute of Physics. 


\title{
Explicit solutions of the classical Calogero and Sutherland systems for any root system
}

\author{
R. Sasaki \\ Yukawa Institute for Theoretical Physics, Kyoto University, Kyoto 606-8502, Japan \\ K. Takasaki \\ Graduate School of Human and Environmental Sciences, Kyoto University, \\ Kyoto 606-8502, Japan
}

(Received 11 October 2005; accepted 30 November 2005; published online 27 January 2006)

Explicit solutions of the classical Calogero (rational with/without harmonic confining potential) and Sutherland (trigonometric potential) systems is obtained by diagonalization of certain matrices of simple time evolution. The method works for Calogero \& Sutherland systems based on any root system. It generalizes the wellknown results by Olshanetsky and Perelomov for the $A$ type root systems. Explicit solutions of the (rational and trigonometric) higher Hamiltonian flows of the integrable hierarchy can be readily obtained in a similar way for those based on the classical root systems. (C) 2006 American Institute of Physics.

[DOI: $10.1063 / 1.2162334]$

\section{INTRODUCTION}

The classical and quantum integrability/solvability of Calogero-Moser systems ${ }^{1-5}$ manifests itself in many guises; the existence of Lax pairs and/or Dunkl operators, algebraic linearization, quadratic algebras, associated integrable spin chains, "quantized" classical spectra, etc. Among them, a most intuitive understanding of solvability/integrability is provided by the fact that explicit solutions of the classical equations of motion are obtained by diagonalization of certain matrices having trivial time evolution, as shown by Olshanetsky and Perelomov ${ }^{6}$ for the rational and trigonometric potential cases. ${ }^{7}$ Their results are for the systems based on the $A$-type roots. Here we will show that the same results hold universally for systems based on any root system. To be more precise, for the rational potential (without/with harmonic confining potential) cases, the diagonalization method works for any root system, including the noncrystallographic ones. For the trigonometric and hyperbolic potential cases, we show that the explicit diagonalization method holds for any crystallographic root system based on the universal Lax pair. ${ }^{8}$ A simpler form of explicit diagonalization is provided by the minimal Lax pair, ${ }^{9,8}$ which exists only for those based on the $A$, $D, E_{6}$, and $E_{7}$ root systems. The basic idea of the explicit solution method is very closely related to the notion of algebraic linearization, proved universally for any root system by Caseiro-Françoise-Sasaki. ${ }^{10}$ We will follow the notation of Ref. 10 throughout this paper and Eq. (a.b) of this paper will be cited as (Ia.b). Explicit solutions in terms of diagonalization is readily obtained for the higher (rational and trigonometric) Hamiltonian flows belonging to the integrable hierarchy. This works, however, only for those based on the classical root systems, $A, B, C$, and $D$. The conventional Lax pair in terms of the set of vector weights $(A, C$, and $D)$ or the set of short roots $(B)$ is indispensable.

This paper is organized as follows. In Sec. II, the historical background and the logical structure of the Calogero-Moser systems necessary for the present paper are briefly reviewed. The Hamiltonian and the universal Lax pair with rational potential are introduced. The explicit integration in terms of diagonalization is achieved by relating the Lax pair matrices $L$ and $M$ to a matrix $W$ of the same size with trivial time evolution, $\ddot{W}=0$. Section III is devoted to the explicit solution of the systems with rational plus the harmonic confining potential. In Sec. IV, we show 
the explicit solutions of the Sutherland systems, which have trigonometric/hyperbolic potentials. In Sec. IV A a simple form of explicit diagonalization is obtained by reinterpreting the formulas of the algebraic linearization method developed by Caseiro-Françoise-Sasaki. ${ }^{10}$ This is based on the minimal Lax pair, which exists for those based on $A, D, E_{6}$, and $E_{7}$ root systems. A general treatment of explicit integration of the Sutherland systems in terms of the universal Lax pair is provided in Sec. IV B. This applies to any crystallographic root system. Sections V and VI are devoted to the problem of explicit integration of the higher Hamiltonian flows of the integrable hierarchy. The rational potential case is discussed in Sec. V and the trigonometric case in Sec. VI. The final section is for a summary and comments.

\section{RATIONAL POTENTIAL}

The integrability of the Calogero-Moser systems has a long history. First, various types of the integrable potentials are recognized; starting from the Calogero model ${ }^{1}$ with rational $\left(1 / q^{2}\right)$ plus a harmonic confining $\left(q^{2}\right)$ potential followed by the Sutherland model ${ }^{2}$ with a trigonometric $\left(1 / \sin ^{2} q\right)$ potential. Then the pure rational potential $\left(1 / q^{2}\right)^{3}$ and the hyperbolic $\left(1 / \sinh ^{2} q\right)^{4}$ and the elliptic $[\wp(q)]$ potentials $^{5}$ are added to the list of the integrable potentials. As seen in many other subjects in mathematical physics, the quantum groups, the integrable spin chains, YangBaxter equations, etc., the elliptic case is the generic one, giving all the rest in various degenerations. However, each degenerate case, the rational, trigonometric, and the hyperbolic, has its own special properties and merits not shared by the more generic ones; for example, the algebraic linearization ${ }^{10}$ of the degenerate Calogero-Moser systems and the quadratic algebras ${ }^{11}$ for the quantum systems with the superintegrable rational $\left(1 / q^{2}\right)$ potential. In the present article we deal with one of such properties of the degenerate Calogero-Moser systems and it is in fact very closely related to the algebraic linearization. ${ }^{10}$ Second, the nature of the multiparticle interactions of the Calogero-Moser systems is recognized to be governed by the root systems associated with finite reflection (Coxeter/Weyl) groups. ${ }^{12,13}$ The original models ${ }^{1-5}$ are all based on the $A$-type root system related to the symmetric group $S_{N}$, with $N$ being the number of the particles. The $S_{N}$ is also the Weyl group of the special unitary group $S U(N)$. The integrability (the Lax pair) of the systems based on the classical root systems $(A, B, C$, and $D)$ is noticed immediately by Olshanetsky and Perelomov, ${ }^{12,13}$ but the actual demonstration of the integrability of the Calogero-Moser systems based on the exceptional ${ }^{9,14}$ and noncrystallographic root systems ${ }^{8}$ took more years. The classical universal Lax pair applicable for all types of potentials and for any root system ${ }^{8}$ and the quantum universal Lax pair applicable for all degenerate potentials and for any root system ${ }^{15}$ have been known for some years.

Let us denote by $\Delta$ a root system of rank $r$. It is a finite set of $\mathbf{R}^{r}$ vectors that is invariant under reflections in the hyperplane perpendicular to each vector in $\Delta$. A reflection $s_{\rho}$ in terms of a root $\rho$ is defined by

$$
s_{\rho}(x)=x-\rho\left(\rho^{\vee} \cdot x\right), \quad x \in \mathbf{R}^{r},
$$

in which $\rho^{\vee}=2 \rho / \rho^{2}$. Thus $\Delta$ is characterized by

$$
s_{\rho}(\eta) \in \Delta, \quad \forall \rho, \eta \in \Delta .
$$

The dynamical variables are the coordinates $q_{i} \in \mathbf{R}, i=1, \ldots, r$ and their canonically conjugate momenta $p_{i} \in \mathbf{R}, i=1, \ldots, r$, except for the $A_{r}$ case in the ordinary embedding, in which the number of particles is $r+1$. The Hamiltonian for the classical Calogero-Moser system with the rational potential but without the harmonic confining potential is

$$
\mathcal{H}=\frac{1}{2} p^{2}+\frac{1}{2} \sum_{\rho \in \Delta_{+}} \frac{g_{|\rho|}^{2}|\rho|^{2}}{(\rho \cdot q)^{2}},
$$


in which the real and positive coupling constants $g_{|\rho|}$ are defined on orbits of the corresponding Coxeter group. That is, for the simple Lie algebra cases $g_{|\rho|}=g$ for all roots in simply laced models and $g_{|\rho|}=g_{L}$ for long roots and $g_{|\rho|}=g_{S}$ for short roots in nonsimply laced models. In order to define Lax pair matrices $L$ and $M$, let us choose a set of $\mathbf{R}^{r}$ vectors $\mathcal{R}=\{\alpha, \beta, \ldots$,$\} , \# \mathcal{R}=\mathcal{D}$, permuting under the action of the reflection group:

$$
s_{\rho}(\alpha) \in \mathcal{R}, \quad \forall \alpha \in \mathcal{R}, \quad \forall \rho \in \Delta .
$$

We demand that it consists of a single orbit of the Coxeter group, for irreducibility. Then we define $\mathcal{D} \times \mathcal{D}$ matrices indexed by the elements of $\mathcal{R}$ :

$$
\begin{gathered}
p \cdot \hat{H}: \quad(p \cdot \hat{H})_{\alpha \beta}=(p \cdot \alpha) \delta_{\alpha \beta}, \\
\hat{s}_{\rho}: \quad\left(\hat{s}_{\rho}\right)_{\alpha \beta}=\delta_{\alpha, s_{\rho}(\beta)} .
\end{gathered}
$$

Introduce next the $\mathcal{D} \times \mathcal{D}$ matrices $X, L$, and $M:^{9,8}$

$$
\begin{gathered}
X=i \sum_{\rho \in \Delta_{+}} g_{|\rho|}(\rho \cdot \hat{H}) \frac{1}{(\rho \cdot q)} \hat{s}_{\rho}, \\
L=p \cdot \hat{H}+X \\
M=-\frac{i}{2} \sum_{\rho \in \Delta_{+}} g_{|\rho|} \frac{|\rho|^{2}}{(\rho \cdot q)^{2}} \hat{s}_{\rho},
\end{gathered}
$$

and a diagonal matrix:

$$
Q=q \cdot \hat{H}: \quad(Q)_{\alpha \beta}=(q \cdot \alpha) \delta_{\alpha \beta} .
$$

Here $L$ and $Q$ are Hermitian $L^{\dagger}=L, Q^{\dagger}=Q$, and $M$ is anti-Hermitian $M^{\dagger}=-M$.

As shown in Ref. 10, Eqs. (I2.7a), (I2.7b) the time evolution of the matrix $L$ along the flow of the Hamiltonian (2.3) displays the following equations:

$$
\begin{gathered}
\frac{\partial L}{\partial t}=[L, M], \\
\frac{\partial Q}{\partial t}=[Q, M]+L .
\end{gathered}
$$

Next let us define another $\mathcal{D} \times \mathcal{D}$ unitary matrix $U(t)$ by the linear equation and the initial condition:

$$
\frac{\partial U}{\partial t}=U M, \quad U(0)=1_{\mathcal{D}},
$$

in which $1_{\mathcal{D}}$ is the $\mathcal{D} \times \mathcal{D}$ unit matrix. The final step is the introduction of $W(t)$ :

$$
W(t) \equiv U(t) Q(t) U^{-1}(t)
$$

which has a simple time evolution 


$$
\begin{gathered}
\dot{W}=U(\dot{Q}-[Q, M]) U^{-1}=U L U^{-1}, \\
\ddot{W}=U(\dot{L}-[L, M]) U^{-1}=0 .
\end{gathered}
$$

The solution is

$$
W(t)=W(0)+t \dot{W}(0)
$$

with the initial values

$$
W(0)=Q(0), \quad \dot{W}(0)=L(0),
$$

which are determined by the initial values of the canonical variables $q_{j}(0), p_{j}(0), j=1, \ldots, r$. Due to the defining relation of $W(t)$ in terms of the diagonal matrix $Q(t)(2.14)$, the solution $\{q(t)\}$ of the canonical equations of motion,

$$
\frac{\partial q_{j}}{\partial t}=\frac{\partial \mathcal{H}}{\partial p_{j}}, \quad \frac{\partial p_{j}}{\partial t}=-\frac{\partial \mathcal{H}}{\partial q_{j}}, \quad j=1, \ldots, r,
$$

with the above Hamiltonian (2.3), is simply obtained by diagonalizing the above matrix solution (2.17). The conjugate momenta $\{p(t)\}$ are obtained by differentiation $p_{j}(t)=\partial q_{j}(t) / \partial t$.

As promised, this is the universal proof applicable for any root system including the noncrystallographic one. The spectrum of $W(t)(2.17)$ is highly constrained, since its dimension $\mathcal{D}$ is usually much greater than the degree of freedom $r$. The high symmetry of the spectrum is guaranteed by the Coxeter invariance of the theory:

$$
\begin{gathered}
\mathcal{H}\left(s_{\rho}(p), s_{\rho}(q)\right)=\mathcal{H}(p, q), \quad \forall \rho \in \Delta, \\
L\left(s_{\rho}(p), s_{\rho}(q)\right)=\hat{s}_{\rho} L(p, q) \hat{s}_{\rho}, \quad M\left(s_{\rho}(q)\right)=\hat{s}_{\rho} M(q) \hat{s}_{\rho} .
\end{gathered}
$$

The original proof of the explicit integration of the $A$ type systems by Olshanetsky and Perelomov ${ }^{6}$ is the very special case in which the spectrum of $W(t)(2.17)$ is not constrained. Our proof reduces to that of Ref. 6 when $\Delta=A_{r}$ and the set of vector weights is chosen as $\mathcal{R}=\mathbf{V}$, $\# \mathcal{R}=\mathcal{D}=r+1$.

\section{RATIONAL WITH HARMONIC CONFINING POTENTIAL}

The Hamiltonian is now

$$
\mathcal{H}_{\omega}=\frac{1}{2} p^{2}+\frac{1}{2} \omega^{2} q^{2}+\frac{1}{2} \sum_{\rho \in \Delta_{+}} \frac{g_{|\rho|}^{2}|\rho|^{2}}{(\rho \cdot q)^{2}} .
$$

With the same matrices introduced above in the preceding section, the time evolution displays (I3.2a), (I3.2b):

$$
\begin{gathered}
\dot{L}=[L, M]-\omega^{2} Q, \\
\dot{Q}=[Q, M]+L .
\end{gathered}
$$

With the same definition of the unitary matrix $U(t)$ as above (2.13), the matrix

$$
W(t) \equiv U(t) Q(t) U^{-1}(t)
$$

evolves harmonically in time: 


$$
\begin{aligned}
& \dot{W}=U(\dot{Q}-[Q, M]) U^{-1}=U L U^{-1} \\
& \ddot{W}=U(\dot{L}-[L, M]) U^{-1}=-\omega^{2} W .
\end{aligned}
$$

The solution is

$$
W(t)=\cos \omega t W(0)+\omega^{-1} \sin \omega t \dot{W}(0),
$$

with the initial values

$$
W(0)=Q(0), \quad \dot{W}(0)=L(0) .
$$

Again the explicit solution $\{q(t)\}$ is obtained by diagonalizing the above matrix $W(t)(3.7)$ with the harmonic time dependence.

\section{TRIGONOMETRIC POTENTIAL}

The Hamiltonian of the trigonometric (Sutherland) model $^{2}$ is written as:

$$
\mathcal{H}=\frac{1}{2} p^{2}+\frac{1}{2} \sum_{\rho \in \Delta_{+}} \frac{g_{|\rho|}^{2}|\rho|^{2}}{\sin ^{2}(\rho \cdot q)} .
$$

In order to get the hyperbolic case, it suffices to change sin into sinh. In the following, we only demonstrate the explicit integration of the trigonometric case. The hyperbolic case can be deduced easily by the above replacement.

Two types of Lax pairs are known ${ }^{9,8}$ for the trigonometric cases: the minimal and the universal Lax pairs. While the latter, the universal lax pair, applies to any crystallographic root system, the former, the minimal Lax pair, requires $\mathcal{R}$ to be the set of minimal weights, which exists only for the $A, D, E_{6}$, and $E_{7}$ root systems. Let us start with the minimal Lax pair, which has a simpler structure thanks to the restriction to the minimal weights, satisfying the condition

$$
\mu \text { : minimal weight } \Leftrightarrow \alpha^{\vee} \cdot \mu=0, \pm 1, \quad \forall \alpha \in \Delta \text {. }
$$

\section{A. Minimal Lax pair}

We consider the matrices ${ }^{9,8}$

$$
\begin{gathered}
L=p \cdot \hat{H}+X, \\
X=i \sum_{\rho \in \Delta_{+}} g_{|\rho|}(\rho \cdot \hat{H}) \frac{1}{\sin (\rho \cdot q)} \hat{s}_{\rho} \\
M=-\frac{i}{2} \sum_{\rho \in \Delta_{+}} g_{|\rho|} \frac{|\rho|^{2} \cos (\rho \cdot q)}{\sin ^{2}(\rho \cdot q)}\left(\hat{s}_{\rho}-1_{\mathcal{D}}\right)+i \sum_{\rho \in \Delta_{+}} g_{|\rho|} \frac{(\rho \cdot \hat{H})^{2}}{\sin ^{2}(\rho \cdot q)},
\end{gathered}
$$

and diagonal matrices:

$$
\begin{gathered}
Q=q \cdot \hat{H}: \quad(Q)_{\alpha \beta}=(q \cdot \alpha) \delta_{\alpha \beta}, \\
R=\mathrm{e}^{2 i Q} .
\end{gathered}
$$

Again $L$ and $Q$ are Hermitian $L^{\dagger}=L, Q^{\dagger}=Q$ and $M$ is anti-Hermitian $M^{\dagger}=-M$. Thus $R$ is unitary. 
As shown in Ref. 10 (I5.3a), (I5.3b), when the root system admits a minimal representation, and $\mathcal{R}$ being the set of minimal weights, the time evolution along the flow of the Hamiltonian (4.1) displays

$$
\begin{gathered}
\frac{\partial L}{\partial t}=[L, M], \\
\frac{\partial R}{\partial t}=[R, M]+i(R L+L R) .
\end{gathered}
$$

With the same definition of the unitary matrix $U(t)$ as above (2.13),

$$
\frac{\partial U}{\partial t}=U M, \quad U(0)=1_{\mathcal{D}}
$$

we introduce a matrix

$$
\mathcal{W}(t)=U(t) R(t) U(t)^{-1}=U(t) \mathrm{e}^{2 i Q(t)} U(t)^{-1} .
$$

It satisfies a simple first-order linear differential equation,

$$
\begin{gathered}
\frac{\partial \mathcal{W}}{\partial t}=U(\partial R / \partial t-[R, M]) U^{-1}=i U(R L+L R) U^{-1} \\
=i\left(\mathcal{W} U L U^{-1}+U L U^{-1} \mathcal{W}\right),
\end{gathered}
$$

since as in (2.15), (2.16), $U L U^{-1}$ is a constant matrix:

$$
\begin{gathered}
\frac{\partial}{\partial t}\left(U L U^{-1}\right)=U(\partial L / \partial t-[L, M]) U^{-1}=0, \\
U(t) L(t) U(t)^{-1}=L(0) .
\end{gathered}
$$

The solution is

$$
\mathcal{W}(t)=\mathrm{e}^{i t L(0)} \mathrm{e}^{2 i Q(0)} \mathrm{e}^{i t L(0)}
$$

By diagonalizing the above matrix solution, we obtain the explicit solution $\{q(t)\}$ of the classical Sutherland model (4.1). One might naturally wonder if the coordinates $\{q(t)\}$ could be determined uniquely from the unitary matrix (4.16). The answer is affirmative since the motion is always restricted to one of the Weyl alcoves due to the periodicity and singularity of the potential. Near the boundary of a Weyl alcove, for example, at $\rho \cdot q=0, \rho \in \Delta$, the singularity of the potential $\sim 1 /(\rho \cdot q)^{2}$ can never be surpassed classically. Therefore if $\{q(0)\}$ is in the principal Weyl alcove,

$$
P W_{T}=\left\{q \in \mathbf{R}^{r} \mid \rho \cdot q>0, \quad \rho \in \Pi, \quad \rho_{h} \cdot q<\pi\right\},
$$

$\{q(t)\}$ will always remain there. Here $\Pi$ is the set of the simple roots and $\rho_{h}$ is the highest weight. This removes any ambiguity in determining $\{q(t)\}$ from the eigenvalues of (4.16). As in the rational potential cases, the spectrum of $\mathcal{W}(t)$ is highly constrained as a consequence of the Weyl invariance (2.20), (2.21).

\section{B. Universal Lax pair}

The universal Lax pair has $\cot (\rho \cdot q)$ function in $L$ instead of $1 / \sin (\rho \cdot q)$ in (4.4),

$$
L=p \cdot \hat{H}+X
$$




$$
\begin{gathered}
X=i \sum_{\rho \in \Delta_{+}} g_{|\rho|}(\rho \cdot \hat{H}) \cot (\rho \cdot q) \hat{s}_{\rho}, \\
M=-\frac{i}{2} \sum_{\rho \in \Delta_{+}} g_{|\rho|} \frac{|\rho|^{2}}{\sin ^{2}(\rho \cdot q)}\left(\hat{s}_{\rho}-1_{D}\right),
\end{gathered}
$$

which satisfy $\partial L / \partial t=[L, M]$ for the Hamiltonian flow, but the additional equation (4.9) takes a different form.

For $\mathcal{R}$ being the set of minimal weights, it reads as

$$
\frac{\partial R}{\partial t}=[R, M]+i(R(L+K)+(L-K) R),
$$

in which $K$ is a non-negative constant matrix commuting with $M$ :

$$
K \equiv \sum_{\rho \in \Delta_{+}} g_{|\rho|}(\rho \cdot \hat{H})\left(\rho^{\vee} \cdot \hat{H}\right) \hat{s}_{\rho}, \quad[K, M]=0 .
$$

It is a very important quantity in Calogero-Moser systems appearing in many contexts. For example, it is a commutator of $Q$ (2.10) and the rational Lax matrix $L(2.8),(2.7)$ [see (4.36) of Ref. 15 and (2.40) of Ref. 16):

$$
[Q, L]=i K
$$

It should be noted that if $K$ is defined as above, the expression (4.22) is universal, which is valid for any root system $\Delta$ and any choice of $\mathcal{R}$. Various properties of the $K$ matrix, whose eigenvalues are all "integers," are discussed in detail by Corrigan-Sasaki, in the Appendix of Ref. 16.

For $\mathcal{R}$ being the set of all roots $\Delta$ (for the simply laced root systems) or the set of short roots $\Delta_{S}$ (for nonsimply laced root systems) and also the set of vector weights $(\mathbf{V})$ for the $C$, the relation corresponding to (4.9) and (4.21) reads as

$$
\frac{\partial R}{\partial t}=[R, M]+i(R(L+\widetilde{K})+(L-\tilde{K}) R),
$$

in which $\widetilde{K}$ is another constant matrix commuting with $M$,

$$
\widetilde{K}=\sum_{\rho \in \Delta_{+}} g_{|\rho|}|\rho \cdot \hat{H}| \hat{s}_{\rho}, \quad[\tilde{K}, M]=0,
$$

introduced by Corrigan-Sasaki, as (5.32) of Ref. 16.

Now the explicit solution of the Sutherland system is achieved for any crystallographic root system, since one can choose at least one Lax pair satisfying (4.24). We proceed as before by defining the unitary matrix $U(t)$ by (4.10) and introduce a matrix

$$
\mathcal{W}(t)=U(t) R(t) U(t)^{-1}=U(t) \mathrm{e}^{2 i Q(t)} U(t)^{-1} .
$$

It satisfies a simple first-order linear differential equation,

$$
\begin{gathered}
\frac{\partial \mathcal{W}}{\partial t}=U(\partial R / \partial t-[R, M]) U^{-1}=i U(R(L+\widetilde{K})+(L-\widetilde{K}) R) U^{-1}, \\
=i\left(\mathcal{W} U(L+\widetilde{K}) U^{-1}+U(L-\widetilde{K}) U^{-1} \mathcal{W}\right),
\end{gathered}
$$

since as in (2.15), (2.16), $U(L \pm \widetilde{K}) U^{-1}$ is a constant matrix: 


$$
\begin{gathered}
\frac{d}{d t}\left(U(L \pm \widetilde{K}) U^{-1}\right)=U(\partial L / \partial t-[L, M]) U^{-1}=0, \\
U(t)(L(t) \pm \widetilde{K}) U(t)^{-1}=L(0) \pm \widetilde{K}
\end{gathered}
$$

The solution is

$$
\mathcal{W}(t)=\mathrm{e}^{i t(L(0)-\tilde{K})} \mathrm{e}^{2 i Q(0)} \mathrm{e}^{i t(L(0)+\tilde{K})} .
$$

By diagonalizing the above matrix solution, we obtain the explicit solution $\{q(t)\}$ of the classical Sutherland system (4.1) for any root system.

The very fact that $\widetilde{K}(K)$ commutes with $M$ simply means that a spectral parameter $\lambda$ can be introduced trivially into the Lax pair for degenerate potentials: ${ }^{8}$

$$
L_{\lambda} \equiv L+\lambda \tilde{K}, \quad \dot{L}_{\lambda}=\left[L_{\lambda}, M\right] .
$$

\section{RATIONAL HIGHER FLOWS}

The integrable hierarchy of the Calogero-Sutherland systems consists of Hamiltonians generated by higher conserved quantities, which are constructed, for example, from the trace of the higher powers of the $L$ matrix, $\mathcal{H}_{n} \propto \operatorname{Tr}\left(L^{2 n}\right)$. The method of explicit integration as described in the preceding sections applies also to these higher Hamiltonian flows, as shown by Suris ${ }^{7}$ for the $A$-type root systems with the conventional Lax pair, $\mathcal{R}=\mathbf{V}$. However, in contrast to the basic Calogero-Sutherland flows, it works only for those systems based on the classical root systems, the $A, B, C$, and $D$. Let us denote by $\mathcal{R}$ the set of vector weights $\mathbf{V}$, for the $A, C$, and $D$ root systems and the set of short roots $\Delta_{S}$ for the $B$ root system. These particular sets $\mathcal{R}$ have a unique orthogonality property,

$$
\text { if } \mu \neq \pm \nu, \quad \mu \cdot \nu=0, \quad \forall \mu, \nu \in \mathcal{R},
$$

which endows a very special structure to the Lax pair represented on $\mathcal{R}$. The dimensions of the corresponding Lax matrices are $\mathcal{D}=r+1$ for the $A_{r}$ and $\mathcal{D}=2 r$ for the $B_{r}, C_{r}$, and $D_{r}$. It is through these special Lax matrices that the explicit integration of the higher rational and trigonometric flows is realized.

Let us start with the explicit forms of the rational $L$ matrices:

$$
\begin{gathered}
(A): L, \quad L_{j k}=p_{j} \delta_{j k}+i g\left(1-\delta_{j k}\right) /\left(q_{j}-q_{k}\right), \\
(B): L=\left(\begin{array}{cc}
A & B \\
-B & -A
\end{array}\right), \quad A_{j k}=p_{j} \delta_{j k}+i g_{L}\left(1-\delta_{j k}\right) /\left(q_{j}-q_{k}\right), \\
B_{j k}=i\left(g_{S} / q_{j}\right) \delta_{j k}+i g_{L}\left(1-\delta_{j k}\right) /\left(q_{j}+q_{k}\right) .
\end{gathered}
$$

The rational $C$ system will not be discussed since it is equivalent to the rational $B$ system. The rational $D$ system is obtained by constraining $g_{S}=0$ in the rational $B$ system.

The higher Hamiltonians are

$$
\begin{aligned}
& (A): \mathcal{H}_{n}=\operatorname{Tr}\left(L^{n+1}\right) /(n+1), \quad n \geqslant 1, \\
& (B, D): \mathcal{H}_{n}=\operatorname{Tr}\left(L^{2 n}\right) /(4 n), \quad n \geqslant 1 .
\end{aligned}
$$

The lowest $\mathcal{H}_{1}$ is the original Hamiltonian (2.3). The basic idea is to rewrite the Hamiltonian flow, 


$$
\frac{\partial q_{j}}{\partial t_{n}}=\frac{\partial \mathcal{H}_{n}}{\partial p_{j}}, \quad \frac{\partial p_{j}}{\partial t_{n}}=-\frac{\partial \mathcal{H}_{n}}{\partial q_{j}}
$$

into equivalent matrix forms,

$$
\begin{gathered}
\frac{\partial L}{\partial t_{n}}=\left[L, M_{n}\right], \\
\frac{\partial Q}{\partial t_{n}}=\left[Q, M_{n}\right]+L^{n}\left(L^{2 n-1}\right),
\end{gathered}
$$

as in the lowest flow (2.11), (2.12).

In contrast to the lowest flow case in which the explicit form of $M$ is given (2.9), we can interpret part of (5.7) and (5.8) as determining $M_{n}$. The diagonal part of the $Q$ equation (5.8) is equivalent to the first half of the canonical equations (5.6). The off-diagonal part of the $Q$ equation (5.8) determines the off-diagonal part of $M_{n}$ completely:

$$
\left(M_{n}\right)_{\mu \nu}=-\left(L^{n}\right)_{\mu \nu} / q \cdot(\mu-\nu), \quad\left(-\left(L^{2 n-1}\right)_{\mu \nu} / q \cdot(\mu-\nu)\right), \quad \mu \neq \nu .
$$

Whereas the diagonal part of $M_{n}$ does not enter the $Q$ equation (5.8), it can be determined from the off-diagonal part of the Lax equation. The result is very simple:

$$
\left(M_{n}\right)_{\mu \mu}=-\sum_{\nu \neq \mu}\left(M_{n}\right)_{\nu \mu}=-\sum_{\nu \neq \mu}\left(M_{n}\right)_{\mu \nu}, \quad M_{n}^{\dagger}=-M_{n} .
$$

The proof that the diagonal part of the higher flow Lax equation (5.7) is equivalent to the second half of the canonical equations (5.6) goes almost parallel to that of the lowest flow.

After the equivalence of the canonical equations (5.6) with the two matrix equations (5.7) and (5.8) is established, the explicit integration by diagonalization is straightforward. Let us define a $\mathcal{D} \times \mathcal{D}$ unitary matrix $U_{n}\left(t_{n}\right)$ by the linear equation and the initial condition:

$$
\frac{\partial U_{n}}{\partial t_{n}}=U_{n} M_{n}, \quad U_{n}(0)=1_{\mathcal{D}} .
$$

Then a matrix function $W_{n}\left(t_{n}\right)$, defined by

$$
W_{n}\left(t_{n}\right) \equiv U_{n}\left(t_{n}\right) Q\left(t_{n}\right) U_{n}^{-1}\left(t_{n}\right),
$$

has a simple time evolution,

$$
\begin{gathered}
\frac{\partial W_{n}}{\partial t_{n}}=U_{n}\left(\partial Q / \partial t_{n}-\left[Q, M_{n}\right]\right) U_{n}^{-1}=\left(U_{n} L U_{n}^{-1}\right)^{n} \quad\left(\left(U_{n} L U_{n}^{-1}\right)^{2 n-1}\right) \\
\frac{\partial}{\partial t_{n}}\left(U_{n} L U_{n}^{-1}\right)=U_{n}\left(\partial L / \partial t_{n}-\left[L, M_{n}\right]\right) U_{n}^{-1}=0 \\
\Rightarrow U_{n}\left(t_{n}\right) L\left(t_{n}\right) U_{n}\left(t_{n}\right)^{-1}=L(0)
\end{gathered}
$$

The solution is

$$
W_{n}\left(t_{n}\right)=W_{n}(0)+t_{n} \partial W_{n}(0) / \partial t_{n},
$$

with the initial values

$$
W_{n}(0)=Q(0), \quad \partial W_{n}(0) / \partial t_{n}=L(0)^{n} \quad\left[L(0)^{2 n-1}\right],
$$

which are determined by the initial values of the canonical variables $q_{j}(0), p_{j}(0), j=1, \ldots, r$. Due 
to the defining relation of $W_{n}\left(t_{n}\right)$ in terms of the diagonal matrix $Q\left(t_{n}\right)(5.12)$, the solution $\left\{q\left(t_{n}\right)\right\}$ of the canonical equations of motion (5.6) with the above Hamiltonian (5.4) or (5.5), is simply obtained by diagonalizing the above matrix solution (5.16). Determination of the conjugate momenta $\left\{p\left(t_{n}\right)\right\}$ requires a solution of the second half of the canonical equations of motion (5.6), which are now algebraic since $\left\{\partial q / \partial t_{n}\right\}$ are now known functions of time. An extension to the generic higher flows of the hierarchy

$$
\mathcal{H}=\sum_{n} c_{n} \mathcal{H}_{n}, \quad c_{n}: \text { const }
$$

is straightforward since the matrix equations (5.7) and (5.8) are linear in $M_{n}$. However, some higher flows cannot be treated this way. For example, in the $D_{r}(r$ :odd) theory, there exists another conserved quantity (Hamiltonian) of the form $p_{1} p_{2} \cdots p_{r}+\cdots$, which cannot be written as (5.18).

\section{TRIGONOMETRIC HIGHER FLOWS}

The basic logics of the explicit integration of the trigonometric higher flows is almost the same as that of the rational higher flows, except that we have to consider two different types of Lax pairs; the minimal and the universal. So we just write down the key formulas without a detailed derivation.

\section{A. Minimal Lax pair}

We discuss the explicit integration of the trigonometric higher flows of the $A$ and $D$ theory in terms of the minimal Lax pair, although the formulation in terms of the universal Lax pair works well for them, too.

The explicit forms of the trigonometric minimal $L$ matrices are

$$
\begin{gathered}
(A): L, \quad L_{j k}=p_{j} \delta_{j k}+i g\left(1-\delta_{j k}\right) / \sin \left(q_{j}-q_{k}\right), \\
(D): L=\left(\begin{array}{cc}
A & B \\
-B & -A
\end{array}\right), \quad A_{j k}=p_{j} \delta_{j k}+i g\left(1-\delta_{j k}\right) / \sin \left(q_{j}-q_{k}\right), \\
B_{j k}=i g\left(1-\delta_{j k}\right) / \sin \left(q_{j}+q_{k}\right) .
\end{gathered}
$$

The higher Hamiltonians take exactly the same form as (5.4) and (5.5). The lowest $\mathcal{H}_{1}$ is the original Hamiltonian (4.1). We rewrite the higher Hamiltonian flow (5.6) into equivalent matrix forms,

$$
\begin{gathered}
\frac{\partial L}{\partial t_{n}}=\left[L, M_{n}\right], \\
\frac{\partial R}{\partial t_{n}}=\left[R, M_{n}\right]+i\left(R L^{n}+L^{n} R\right) \quad\left(i\left(R L^{2 n-1}+L^{2 n-1} R\right)\right),
\end{gathered}
$$

as in the lowest flow (4.8), (4.9). The off-diagonal part of $M_{n}$ is

$$
\left(M_{n}\right)_{\mu \nu}=-\left(L^{n}\right)_{\mu \nu} \cot [q \cdot(\mu-\nu)] \quad\left(-\left(L^{2 n-1}\right)_{\mu \nu} \cot [q \cdot(\mu-\nu)]\right), \quad \mu \neq \nu .
$$

The diagonal part is

$$
\left(M_{n}\right)_{\mu \mu}=\sum_{\nu \neq \mu}\left(L^{n}\right)_{\nu \mu} / \sin [q \cdot(\nu-\mu)]=\sum_{\nu \neq \mu}\left(L^{n}\right)_{\mu \nu} / \sin [q \cdot(\mu-\nu)], \quad M_{n}^{\dagger}=-M_{n} .
$$

The $\mathcal{D} \times \mathcal{D}$ matrix $\mathcal{W}_{n}\left(t_{n}\right)$ obeys a simple time evolution: 


$$
\begin{gathered}
\mathcal{W}_{n}\left(t_{n}\right)=U_{n}(t) R\left(t_{n}\right) U_{n}\left(t_{n}\right)^{-1}=U_{n}\left(t_{n}\right) \mathrm{e}^{2 i Q\left(t_{n}\right)} U_{n}\left(t_{n}\right)^{-1}, \\
=\mathrm{e}^{i t_{n} L(0)^{n}} \mathrm{e}^{2 i Q(0)} \mathrm{e}^{i t_{n} L(0)^{n}}\left(\mathrm{e}^{i t_{n} L(0)^{2 n-1}} \mathrm{e}^{2 i Q(0)} \mathrm{e}^{i t_{n} L(0)^{2 n-1}}\right) .
\end{gathered}
$$

By diagonalizing the above matrix solution (6.8), we obtain the explicit solution $\left\{q\left(t_{n}\right)\right\}$ of the higher flows of the Sutherland system (5.4) and (5.5) for the $A$ and $D$ root systems.

\section{B. Universal Lax pair}

The explicit integration of the higher flows of the $B$ and $C$ Sutherland systems is achieved in terms of the universal Lax pairs based on the set of short roots $\left(\mathcal{R}=\Delta_{S}\right)$ for $B$ and the set of vector weights $(\mathcal{R}=\mathbf{V})$ for $C$. For the rank $r$ system, both have $\mathcal{D}=2 r$.

The Lax matrix $L$ and the constant matrix $\widetilde{K}(4.25)$ are

$$
\begin{gathered}
L=\left(\begin{array}{cc}
A & B \\
-B & -A
\end{array}\right), \quad \widetilde{K}=\left(\begin{array}{cc}
S & T \\
T & S
\end{array}\right), \\
(B): A_{j k}=p_{j} \delta_{j k}+i g_{L}\left(1-\delta_{j k}\right) \cot \left(q_{j}-q_{k}\right), \quad S_{j k}=g_{L}\left(1-\delta_{j k}\right), \\
B_{j k}=i g_{S} \cot q_{j} \delta_{j k}+i g_{L}\left(1-\delta_{j k}\right) \cot \left(q_{j}+q_{k}\right), \quad T_{j k}=g_{S} \delta_{j k}+g_{L}\left(1-\delta_{j k}\right), \\
(C): A_{j k}=p_{j} \delta_{j k}+i g_{S}\left(1-\delta_{j k}\right) \cot \left(q_{j}-q_{k}\right), \quad S_{j k}=g_{S}\left(1-\delta_{j k}\right), \\
B_{j k}=2 i g_{L} \cot 2 q_{j} \delta_{j k}+i g_{S}\left(1-\delta_{j k}\right) \cot \left(q_{j}+q_{k}\right), \quad T_{j k}=2 g_{L} \delta_{j k}+g_{S}\left(1-\delta_{j k}\right) .
\end{gathered}
$$

It is easy to see

$$
\begin{gathered}
\mathrm{e}^{i Q}(L+\widetilde{K}) \mathrm{e}^{-i Q}=\mathrm{e}^{-i Q}(L-\widetilde{K}) \mathrm{e}^{i Q}, \\
\Rightarrow \operatorname{Tr}(L+\widetilde{K})^{n}=\operatorname{Tr}(L-\widetilde{K})^{n},
\end{gathered}
$$

which are conserved quantities of the Sutherland flow (4.1). It differs from the usual one $\operatorname{Tr}\left(L^{n}\right)$ by a linear combination of lower-order conserved quantities. The canonical equations of the higher flow Hamiltonian,

$$
\mathcal{H}_{n}=\operatorname{Tr}\left((L \pm \widetilde{K})^{2 n}\right) /(4 n)
$$

are equivalent to the matrix equations

$$
\begin{gathered}
\frac{\partial L}{\partial t_{n}}=\left[L, M_{n}\right], \\
\frac{\partial R}{\partial t_{n}}=\left[R, M_{n}\right]+i\left(R(L+\widetilde{K})^{2 n-1}+(L-\tilde{K})^{2 n-1} R\right) .
\end{gathered}
$$

The off-diagonal part of $M_{n}$ is

$$
\left(M_{n}\right)_{\mu \nu}=-\left[e^{i q \cdot(\mu-\nu)}(L+\widetilde{K})_{\mu \nu}^{n}+e^{-i q \cdot(\mu-\nu)}(L-\tilde{K})_{\mu \nu}^{n}\right] / \sin [q \cdot(\mu-\nu)], \quad \mu \neq \nu .
$$

The diagonal part is 


$$
\left(M_{n}\right)_{\mu \mu}=-\sum_{\nu \neq \mu}\left(M_{n}\right)_{\nu \mu}=-\sum_{\nu \neq \mu}\left(M_{n}\right)_{\mu \nu}, \quad M_{n}^{\dagger}=-M_{n} .
$$

The $\mathcal{D} \times \mathcal{D}$ matrix $\mathcal{W}_{n}\left(t_{n}\right)$ obeys simple time evolution:

$$
\begin{aligned}
\mathcal{W}_{n}\left(t_{n}\right) & =U_{n}(t) R\left(t_{n}\right) U_{n}\left(t_{n}\right)^{-1}=U_{n}\left(t_{n}\right) \mathrm{e}^{2 i Q\left(t_{n}\right)} U_{n}\left(t_{n}\right)^{-1} \\
& =\mathrm{e}^{i t_{n}(L(0)-\tilde{K})^{2 n-1}} \mathrm{e}^{2 i Q(0)} \mathrm{e}^{i t_{n}(L(0)+\tilde{K})^{2 n-1}} .
\end{aligned}
$$

By diagonalizing the above matrix solution (6.22), we obtain the explicit solution $\left\{q\left(t_{n}\right)\right\}$ of the higher flows of the Sutherland system (6.16) for the $B$ and $C$ root systems.

\section{SUMMARY AND COMMENTS}

Explicit integration of the Calogero and Sutherland systems by means of diagonalization is demonstrated for any root system, the exceptional as well as the classical and the noncrystallographic. It is based on the universal Lax pair for the degenerate potentials, which is the rational with/without the harmonic confining potential and the trigonometric/hyperbolic potentials. As emphasized in the text, it is very closely related to the concept of algebraic linearization by Caseiro-Françoise-Sasaki. ${ }^{10}$ The method is extended to the higher Hamiltonian flows of the rational and trigonometric/hyperbolic interactions. In contrast to the basic Calogero-Sutherland flows, the applicability is limited to those systems based on the classical root systems, the $A, B, C$, and $D$ root systems.

The theory of explicit integration of higher Hamiltonian flows is very closely related to the dynamical $r$ matrix $^{7,17,18}$ and the Hamiltonian reduction. ${ }^{19,13}$ In the case of the most classical rational potential of the A type, the method of Hamiltonian reduction starts from the large phase space of the matrix dynamical variable $W$ and its conjugate momentum variable $Z$, which are both assumed to be Hermitian. The Hamiltonians,

$$
\mathcal{H}_{n}=\operatorname{Tr}\left(Z^{n+1}\right) /(n+1),
$$

generate the flows

$$
\frac{\partial W}{\partial t_{n}}=Z^{n}, \quad \frac{\partial Z}{\partial t_{n}}=0 .
$$

This Hamiltonian system is invariant under the action $(W, Z) \rightarrow\left(U W U^{-1}, U Z U^{-1}\right)$ of unitary matrices $U$. The reduced phase space is obtained by imposing the constraint

$$
[W, Z]=i K
$$

and factoring out the constrained phase space by residual symmetries (i.e., by the group of unitary matrices that commute with $K)$. The $(Q, L)$ pair $(4.23)$ is nothing but a representative of a point of the reduced phase space, which is connected with the point $(W, Z)$ of the large phase space by a ( $t$-dependent) unitary matrix $U$ as

$$
Q=U^{-1} W U, \quad L=U^{-1} Z U .
$$

The linear flows of $(W, Z)$ are thereby mapped to the Calogero flows of $(Q, L)$. This is the way to understand the rational Calogero system of the A type as a Hamiltonian reduction; ${ }^{19}$ a similar interpretation has been proposed for a few other cases. ${ }^{13}$ The dynamical $r$ matrix has been constructed in this framework of Hamiltonian reduction. ${ }^{7}$ We expect that all the cases discussed in this paper can be treated in the same way. 


\section{ACKNOWLEDGMENTS}

We thank F. Calogero and J.-P. Françoise for illuminating discussion. We are supported in part by a Grant-in-Aid for Scientific Research from the Ministry of Education, Culture, Sports, Science and Technology, No.16340040.

${ }^{1}$ F. Calogero, "Solution of the one-dimensional $N$-body problem with quadratic and/or inversely quadratic pair potentials," J. Math. Phys. 12, 419-436 (1971).

${ }^{2}$ B. Sutherland, "Exact results for a quantum many-body problem in one-dimension. II," Phys. Rev. A 5, 1372-1376 (1972).

${ }^{3}$ J. Moser, "Three integrable Hamiltonian systems connected with isospectral deformations," Adv. Math. 16, 197-220 (1975); J. Moser, "Integrable systems of non-linear evolution equations", in Dynamical Systems, Theory and Applications, Lecture Notes in Physics 38 (Springer-Verlag, Berlin 1975).

${ }^{4}$ F. Calogero, C. Marchioro, and O. Ragnisco, "Exact solution of the classical and quantal one-dimensional many body problems with the two body potential $V_{a}(x)=g^{2} a^{2} / \sinh ^{2} a x$," Lett. Nuovo Cimento Soc. Ital. Fis. 13, 383-387 (1975).

${ }^{5}$ F. Calogero, "Exactly solvable one-dimensional many body problems," Lett. Nuovo Cimento Soc. Ital. Fis. 13, 411-416 (1975).

${ }^{6}$ M. A. Olshanetsky and A. M. Perelomov, "Explicit solution of the Calogero model in the classical case and geodesic flows on symmetric spaces of zero curvature," Lett. Nuovo Cimento Soc. Ital. Fis. 16, 333-339 (1976); M. A. Olshanetsky and A. M. Perelomov, "Explicit solutions of some completely integrable systems," Lett. Nuovo Cimento Soc. Ital. Fis. 17, 97-101 (1976); F. Calogero: Classical many-body problems amenable to exact treatments, Lecture Notes in Physics Monograph m66 (Springer-Verlag, Berlin, 2001).

${ }^{7}$ Y. B. Suris, "Why are the Ruijsenaars-Schneider and the Calogero-Moser hierarchies governed by the same $r$ matrix?," Phys. Lett. A 225, 253 (1997).

${ }^{8}$ A. J. Bordner, E. Corrigan, and R. Sasaki, "Generalized Calogero-Moser models and universal Lax pair operators," Prog. Theor. Phys. 102, 499 (1999).

${ }^{9}$ A. J. Bordner, E. Corrigan, and R. Sasaki, "Calogero-Moser Models. I-A new formulation," Prog. Theor. Phys. 100, 1107 (1998).

${ }^{10}$ R. Caseiro, J. -P. Françoise, and R. Sasaki, "Algebraic linearization of dynamics of Calogero type for any Coxeter group," J. Math. Phys. 41, 4679 (2000).

${ }^{11}$ R. Caseiro, J. -P. Françoise, and R. Sasaki, "Quadratic algebra associated with rational Calogero-Moser models," J. Math. Phys. 42, 5329 (2001).

${ }^{12}$ M. A. Olshanetsky and A. M. Perelomov, "Completely integrable Hamiltonian systems connected with semisimple Lie algebras," Invent. Math. 37, 93 (1976).

${ }^{13}$ M. A. Olshanetsky and A. M. Perelomov, "Classical integrable finite-dimensional systems related to Lie algebras," Phys. Rep. 71, 314 (1981).

${ }^{14}$ E. D'Hoker and D. H. Phong, "Calogero-Moser Lax pairs with spectral parameter for general Lie algebras," Nucl. Phys. B 530, 537 (1998).

${ }^{15}$ A. J. Bordner, N. S. Manton, and R. Sasaki, "Calogero-Moser models V: Supersymmetry and quantum Lax pair," Prog. Theor. Phys. 103, 463 (2000).

${ }^{16}$ E. Corrigan and R. Sasaki, "Quantum vs classical integrability in Calogero-Moser systems," J. Phys. A 35, 7017 (2002).

${ }^{17}$ J. Avan and M. Talon, "Classical $R$ matrix structure for the Calogero model," Phys. Lett. B 303, 33 (1993).

${ }^{18}$ J. Avan, O. Babelon, and M. Talon, "Construction of the classical $R$ matrices for the Toda and Calogero models," Algebra Anal. 6, 67 (1994).

${ }^{19}$ D. Kadzdan, B. Kostant, and S. Sternberg, "Hamiltonian group actions and dynamical systems of Calogero type," Commun. Pure Appl. Math. 31, 481 (1978). 